\title{
Advances in on-chip photodetection for applications in miniaturized genetic analysis systems
}

\author{
Vijay Namasivayam ${ }^{1,2,4}$, Rongsheng Lin ${ }^{1,2,4}$, Brian Johnson ${ }^{1}$, \\ Sundaresh Brahmasandra ${ }^{1}$, Zafar Razzacki ${ }^{1}$, David T Burke ${ }^{3}$ \\ and Mark A Burns ${ }^{1}$ \\ ${ }^{1}$ Department of Chemical Engineering, The University of Michigan, Ann Arbor, \\ MI 48109-2136, USA \\ ${ }^{2}$ Department of Electrical Engineering and Computer Science, The University of Michigan, \\ Ann Arbor, MI 48109-2136, USA \\ ${ }^{3}$ Department of Human Genetics, The University of Michigan, Ann Arbor, MI 48109-2136, \\ USA \\ E-mail: maburns@umich.edu
}

Received 29 April 2003, in final form 18 July 2003

Published 18 August 2003

Online at stacks.iop.org/JMM/14/81 (DOI: 10.1088/0960-1317/14/1/311)

\begin{abstract}
Microfabrication techniques have become increasingly popular in the development of next generation DNA analysis devices. Improved on-chip fluorescence detection systems may have applications in developing portable hand-held instruments for point-of-care diagnostics. Miniaturization of fluorescence detection involves construction of ultra-sensitive photodetectors that can be integrated onto a fluidic platform combined with the appropriate optical emission filters. We have previously demonstrated integration PIN photodiodes onto a microfabricated electrophoresis channel for separation and detection of DNA fragments. In this work, we present an improved detector structure that uses a PINN ${ }^{+}$photodiode with an on-chip interference filter and a robust liquid barrier layer. This new design yields

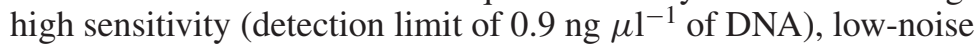
$(\mathrm{S} / \mathrm{N} \sim 100 / 1)$ and enhanced quantum efficiencies $(>80 \%)$ over the entire visible spectrum. Applications of these photodiodes in various areas of DNA analysis such as microreactions (PCR), separations (electrophoresis) and microfluidics (drop sensing) are presented.
\end{abstract}

(Some figures in this article are in colour only in the electronic version)

\section{Introduction}

Development of integrated, portable chemical analysis devices is ultimately limited by the ability to detect low concentrations of labeled DNA detection scheme. The ability to observe DNA in real-time has had significant impact and is the basis for virtually all of the human genomic sequencing performed to date (Hunkapiller et al 1991). Improvements in the basic detection system, such as new fluorescent dyes, continue to influence the overall efficiency of DNA sequencing

4 These authors contributed equally to this work.
(Metzker et al 1996). Recent innovations in miniaturized systems have continued to rely on macroscale, external optical read-out arrangements thus minimizing the benefits of microfabrication (Woolley et al 1998). A miniaturized detection system, with sensitivity comparable to macroscale detection systems, will aid in the realization of integrated 'labon-a-chip' systems.

Most modern sequencing and genetic diagnosis systems employ macroscale rather than microscale detection systems. Fluorescence microscopy is an accurate, time-tested technique and the dyes used to label DNA are extremely efficient, permitting single molecule detection in femtoliter samples 
(Barnes et al 1995). Miniaturization of this technique is not straightforward since it involves the design of ultrasensitive, low noise photodiodes with on-chip filters. Consequently, researchers have investigated the possibility of miniaturizing alternative detection schemes such as electrochemiluminescence and electrochemical detection methods. Miniaturized capillary array electrophoresis devices with end column electrochemical detection (Ewing et al 1994, Woolley etal 1998) and PCR chambers with electrochemiluminescence detection (Northrup et al 1995) have been constructed.

While electrochemiluminescence and electrochemical detection schemes are currently under development, it would be beneficial to explore miniaturization of fluorescence detection method. Fluorescence detection is attractive because it offers high sensitivity, ease of automation and real-time detection of DNA. Reactions such as Sanger sequencing and Polymerase chain reactions have been adapted to fluorescence labeling methods. Capillary electrophoresis systems have been optimized for size-fractionation of DNA fragments labeled with fluorescent dyes. Therefore, developing a sensitive on-chip fluorescence detection system may be useful to carry out a wide variety of biological assays without changing the biochemistry.

We have previously demonstrated the use of an on-chip fluorescence detector for sensing migrating DNA fragments in a microfabricated gel electrophoresis system (Burns et al 1998, Webster et al 2001). In this work, we present an improved diode $\left(\mathrm{n} / \mathrm{n}^{+}\right.$doped) design for detecting DNA samples in low concentrations. The new detector structure is designed for increased fluorescent light absorption in the active region and enhanced photocarrier collection efficiency. The quantum efficiency and hence the sensitivity of the diode is enhanced over the entire visible range of wavelengths. In the following sections, we describe in detail the construction of the photodetector and discuss its applications in several areas of DNA analysis such as microreactions, separations and microfluidics.

\section{Detector design}

Silicon-based PIN photodiodes with on-chip interference filters have been integrated onto electrophoresis columns for detecting fluorescence signals from labeled DNA samples (Burns etal 1998, Webster etal 2001). The detectors presented in these earlier works were developed for DNA band detection in microelectrophoresis columns and could detect double stranded DNA up to $10 \mathrm{ng} \mu \mathrm{l}^{-1}$ concentrations. However, the detector sensitivity would need to be improved in order to sense low concentrations of DNA while performing high-resolution separations or real time PCR.

Diode response to fluorescent signals can be significantly improved (1) by enhancing photocarrier generation in the active region and (2) by increasing the collection efficiency of the generated photocarriers. The first problem is associated with the high light absorption coefficient of silicon and the junction depths in the device. Figure 1 shows the light absorption coefficient $\alpha(\lambda)$ for silicon (Wilson 1988). Since most blue/green dyes label fluoresce in the $500-550 \mathrm{~nm}$ region (e.g., SYBR-green 497/520 nm, YOYO-1 491/509 nm), most

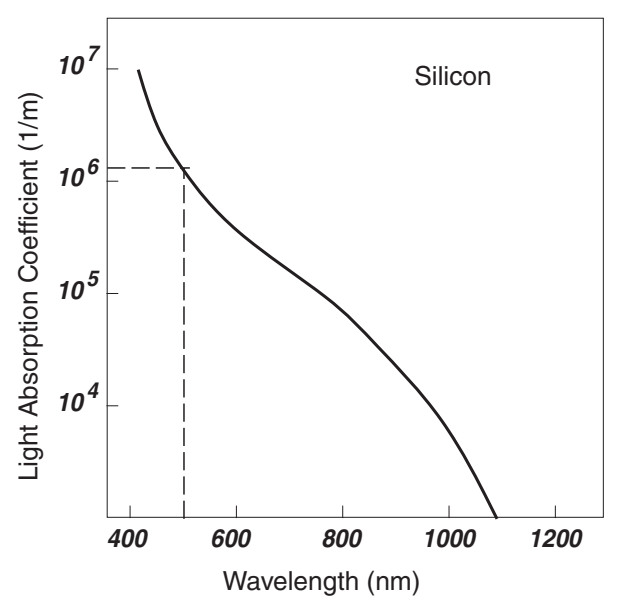

Figure 1. Light absorption coefficient $\alpha\left(\mathrm{m}^{-1}\right)$ for silicon as a function of wavelength. At $500 \mathrm{~nm}$ most of the light is absorbed within $1 \mu \mathrm{m}$ of the surface.

of the emitted fluorescent light is absorbed within $1 \mu \mathrm{m}$ of the oxide interface.

Since the absorption length is so small, we need to be concerned only with the hole generation in the $\mathrm{n}^{+}$region. These carriers must therefore diffuse through the $\mathrm{n}^{+}$region until they reach the edge of the depletion region where they are swept by the field generating a photocurrent. In the structure $\left(\mathrm{n}^{+} \mathrm{p}\right)$ of our earlier device (figure $2(a)$ ), these photocarriers were generated in a heavily doped $\mathrm{n}^{+}$region (junction depth $\sim 0.8 \mu \mathrm{m}$ ) with a large concentration of defects and recombining centers. The characteristic decay length in these heavily doped regions is less than $0.5 \mu \mathrm{m}$ (Chamberlain 1979). Hence, many of the photogenerated holes were lost due to recombination.

The second difficulty is associated with the doping gradients near the junction region. These gradients induce an electric field in the device,

$$
E_{x}=-\frac{k T}{q} \frac{1}{n(x)} \frac{\mathrm{d} n(x)}{\mathrm{d} x}
$$

where $n$ is the effective concentration. The value $n$ is determined by the actual impurity profile and its ability to ionize non-degeneratively. It has been shown that for a Gaussian impurity profile peaked at the surface, the electron concentration $n(x)$ is approximately a Gaussian centered at $x_{0}>0$ and straggle $\sigma$

$$
n(x) \sim N_{0} \mathrm{e}^{-\left(x-x_{0}\right)^{2} / 2 \sigma^{2}} .
$$

Both $x_{0}$ and $\sigma$ depend on the diffusion dose. This electron concentration yields a field

$$
E_{x}=\frac{k T}{q} \frac{\left(x-x_{0}\right)}{\sigma^{2}} .
$$

At any given position $x$, the photocarrier flux is

$$
J=-D \frac{\mathrm{d} p(x)}{\mathrm{d} x}+\mu p E_{x}
$$

where $p(x)$ is the hole concentration. Therefore, the hole flux decreases when $x<x_{0}$ and increases for $x>x_{0}$. In a deep heavily doped profile the former applies and the field pushes most of the photogenerated holes towards the oxide interface and away from the depletion region, thereby reducing the collection efficiency. Together the combined action of these two effects results in low collection efficiencies of $5-10 \%$. 


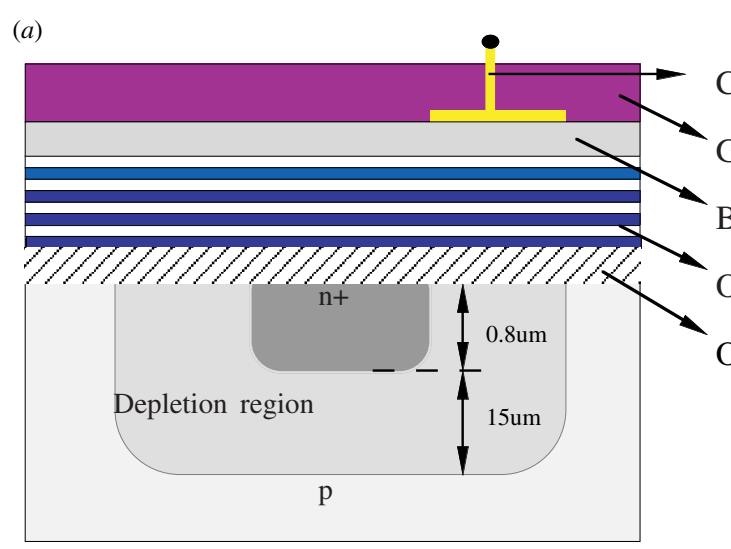

Previous Detector Structure (b)

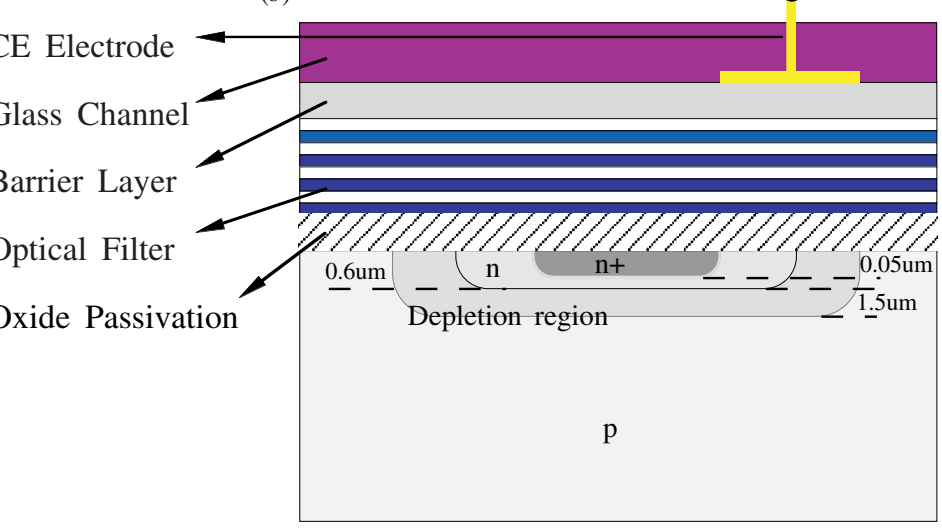

Improved Detector Structure

Figure 2. (a) Cross-section of previous detector structure showing spurious leakage currents and collection inefficiency. (b) Cross-section of improved proposed detector structure showing a $n^{+} n-p$ type of diode structure that increases the diode photocarrier collection efficiency.

In this work, we present a shallow $\mathrm{PINN}^{+}$diode structure (figure $2(b)$ ) in order to improve the detector sensitivity. The junction depths in our new $\mathrm{n}^{+} / \mathrm{n}$ detector structure are designed for $500 \AA(0.05 \mu \mathrm{m})$ of $\mathrm{As}\left(\mathrm{n}^{+}\right)$and $0.6 \mu \mathrm{m}$ of $\mathrm{P}(\mathrm{n})$. With these doping concentrations the depletion region is reduced by an order of magnitude, from $10 \mu \mathrm{m}$ in our earlier design to $1.5 \mu \mathrm{m}$ in the current design. In this design, most of the holes are generated deeper than the $\mathrm{n}^{+}$region, where there is little loss due to recombination. Further, in this structure, collection efficiency is improved as the doping gradient in the $\mathrm{n}^{+}$region pushes the holes towards the depletion region and away from the silicon-oxide interface. The quantum efficiencies for devices with similar structures have been reported to be as high as $85 \%$ for wavelengths up to the UV region (Chamberlain 1979).

\section{Device fabrication}

The process flow for constructing the integrated devices with photodiodes and on-chip interference filter is shown in figure 3 . Our DNA analysis devices consist of two sides:

(1) a silicon side which includes the heaters/temperature sensors and the photodetectors;

(2) a glass side which has the fluidic network. The glass side is glued on to the silicon side to render an integrated microfluidic device. Several photodetectors can thus be incorporated very close to the microfluidic channel at desired locations.

Device construction begins with fabrication of the photodetector within a crystalline silicon substrate. The photodetectors are positioned at multiple locations across the device. Diodes are constructed by implanting the entire surface of a 1000-2000 $\Omega \mathrm{cm},\langle 100\rangle$, float zone, boron-doped, p-type silicon wafer with phosphorus. The high resistivity is used to increase the depletion region and therefore the efficiency of the diode junction. The wafers are first implanted with a field implant $30 \mathrm{KeV}$ boron dose $\left(2 \times 10^{12} \mathrm{~cm}^{-2}\right)$. The field implant prevents inversion of the substrate below the metal lines near the surface (the high resistivity substrates are easily inverted). An alignment key is next etched onto the silicon surface. The photodiode is constructed in the substrate by using two successive ion implantation ( $n$ and $n^{+}$) steps. The $\mathrm{n}$ implantation is first done over the diode regions with a $180 \mathrm{KeV}$ phosphorous at a dose of $3 \times 10^{13} \mathrm{~cm}^{-2}$ using a photoresist mask. The shallow $\mathrm{n}^{+}$implantation is done over the $\mathrm{n}$ regions with a $25 \mathrm{KeV}$ arsenic at a dose of $5 \times 10^{14} \mathrm{~cm}^{-2}$. For making a contact to the substrate, a p-type implantation is done with $50 \mathrm{KeV}$ boron at a dose of $10^{15} \mathrm{~cm}^{-2}$ using another resist masking step. (This higher dose for the boron implant is to compensate for the segregation of the boron in silicon dioxide. When the silicon dioxide layer is later grown on top of the implanted region, the boron dopant concentration will be higher in the oxide layer than in the silicon.) The photoresist masks from the implantation are stripped using PRS 2000 at $100{ }^{\circ} \mathrm{C}$ followed by a 'piranha' clean $\left(1: 1 \mathrm{H}_{2} \mathrm{SO}_{4}: \mathrm{H}_{2} \mathrm{O}_{2}\right)$. The strip and clean steps are repeated until the resist is completely removed.

A passivation thermal oxide layer $(0.2 \mu \mathrm{m}$ thick $)$ is then grown on the wafer using wet TCA oxide conditions at $900{ }^{\circ} \mathrm{C}$. The temperature of the oxide growth is kept low to limit the diffusion of the dopants. Contacts to the substrate and the diode are made using a wet buffered hydrofluoric acid (BHF) etchant. A short 10-20 s BHF dip is used to clean the contact areas. The wafer is then immediately placed into a vacuum chamber. An $\mathrm{Al}$ metallization followed by a $\mathrm{Ti} / \mathrm{Pt}$ metallization were used to form metal contacts to the diode and the substrate contacts. The diodes are tested using a probestation to ensure that good contact was made to the substrate and junction areas.

Next, the wafer is shipped to Z C \& R Coatings, California for interference filter deposition. The filter is deposited above the diodes to allow the transmission of fluorescent-emission light $(515 \mathrm{~nm})$ from dye and block the short wavelength excitation light $(<500 \mathrm{~nm})$. The filter is $\sim 6 \mu \mathrm{m}$ thick and consists of 40 alternating layers of $\mathrm{SiO}_{2}$ and $\mathrm{TiO}_{2}$ thin films. A passivation layer of $1.5 \mu \mathrm{m}$ low temperature oxide or $2.5 \mu \mathrm{m}$ Parylene $\mathrm{C}$ is then deposited over the filter to isolate the fluidic components from the electronic components. The metal heaters and temperature sensors $(500 \AA \mathrm{Ti} / 1000 \AA \mathrm{Pt})$ required for temperature control are fabricated over this passivation layer. Another passivation layer of $1.5 \mu \mathrm{m}$ low temperature 

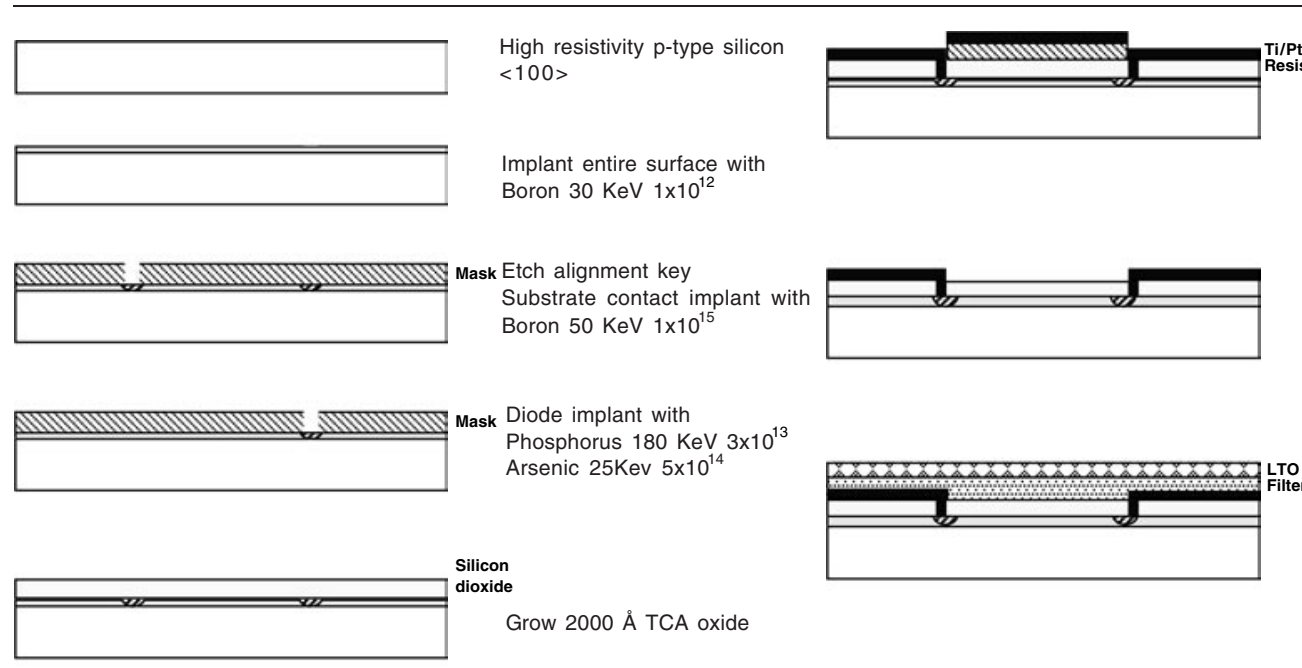

Pt Metal deposition for diode and substrate contacts Deposit Ti/Pt 500/1000Å

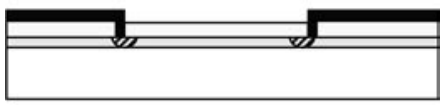

Liftoff
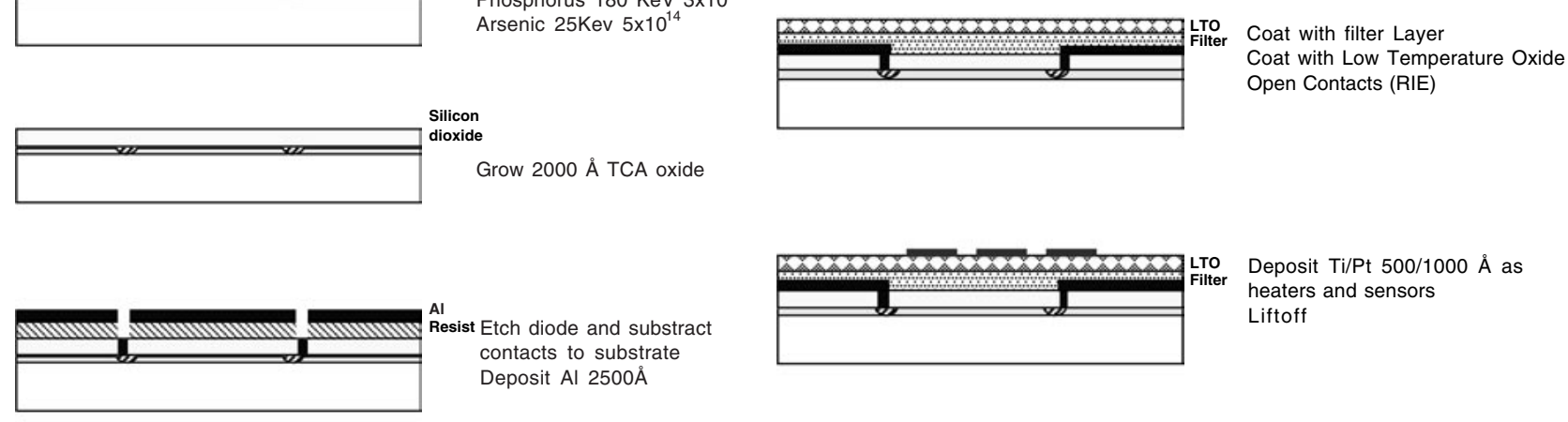

Al

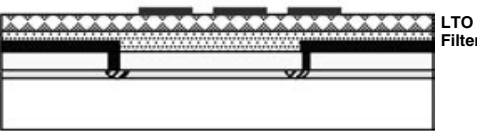

Deposit Ti/Pt 500/1000 Å as heaters and sensors

Etch diode and substract contacts to substrate Deposit Al $2500 \AA$
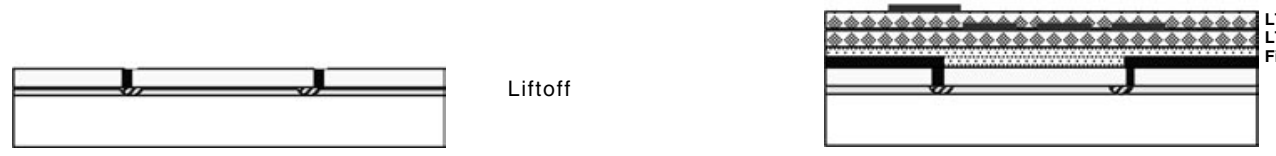

Coat with LTO

Liftoff

(a)

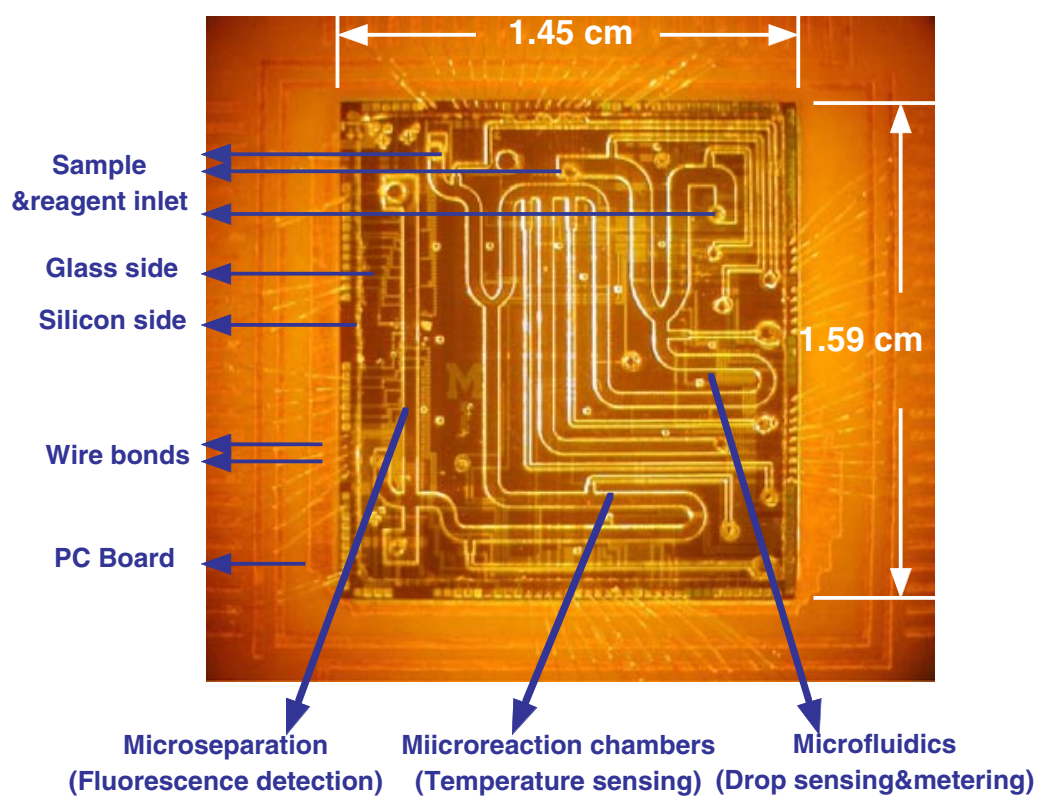

(b)

Figure 3. (a) Process flow for the fabrication of on-chip fluorescence detector. (b) Photograph of a fully constructed and assembled DNA analysis device. Photodiodes are embedded in the integrated device that includes a microreactor, a microseparation column and fluidic channels.

oxide or $2.5 \mu \mathrm{m}$ Parylene $\mathrm{C}$ is then deposited over the heaters and temperature sensors and finally titanium/platinum electrodes $(500 \AA / 1000 \AA)$ are placed at defined locations for use in electrophoresis using a 'lift-off' process.

Fabrication of the glass channels involves a similar process. First metal layers of $600 \AA$ chromium followed by a $4000 \AA$ gold are deposited on the surface of a borofloat glass wafer $(500 \mu \mathrm{m}$ thick, $100 \mathrm{~mm}$ diameter $)$, which had been thermally annealed to provide smooth etched channel sidewalls. A positive photoresist (Microposit SC 1827; Shipley Company, Marlborough, MA, USA) is spin-coated and patterned using a channel mask and developed. The 


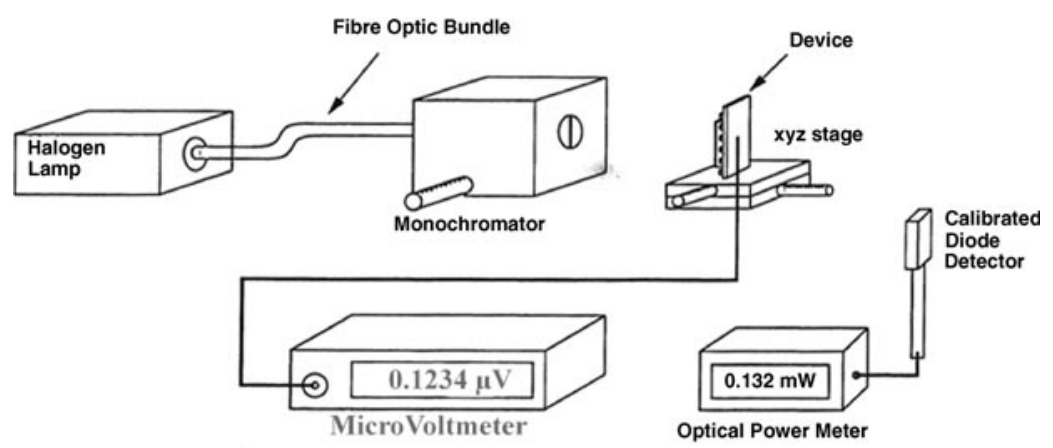

(a)

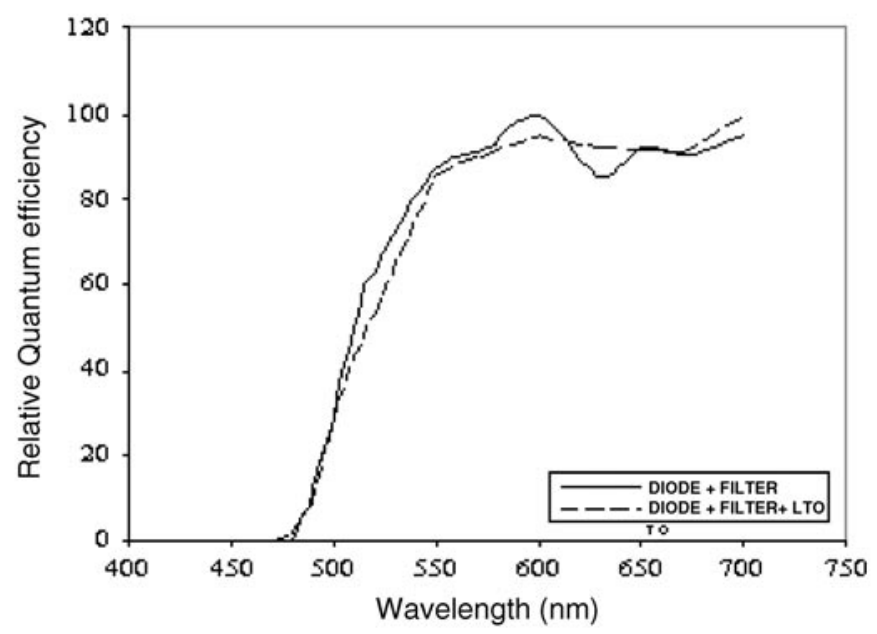

(b)

Figure 4. (a) Experimental setup for measuring the spectral response of the on-chip photodiode + filter combination. (b) Spectral response of the photodiode with $(a)$ on-chip filter, $(b)$ on-chip filter and a $3 \mu \mathrm{m}$ liquid barrier layer (low temperature oxide-LTO).

metal layers are etched in a commercial gold etchant (Gold Etchant TFA, Transene Co.) and chromium etchant (CR-14, Cyantek Corp., Fremont, CA) respectively, leaving glass exposed in the locations where the channel network is to be etched. The accessible glass is then etched in a freshly prepared solution of hydrofluoric acid (49\% HF, CMOS grade; JT Baker). The glass side is then aligned on top of the silicon side and bonded using optical adhesive (SK-9 Lens Bond, Summers Laboratories, Fort Washington, PA). The bond was cured under a UV light source $(365 \mathrm{~nm})$ for $24 \mathrm{~h}$. It is wired to a printed circuit board and fitted with silicone electrophoresis buffer wells.

\section{Results and discussions}

\subsection{Detector characterization}

4.1.1. Filter performance. Fluorescently labeled DNA in the microchannel absorbs the excitation light (from a blue LED) and emits fluorescent light at a slightly longer wavelength (green). To prevent the excitation light from saturating the detector, a thin film, high-pass, interference filter is deposited over the detector surface. The filter was designed to block essentially all wavelengths less than $500 \mathrm{~nm}$, but to allow the fluorescent emission wavelengths $(>500 \mathrm{~nm})$ to pass through. The filter efficiency can be defined as the ratio of the transmitted fluorescent light to the transmitted excitation light.
Based on our tests, we find that the filter has 5\% transmittance at $490 \mathrm{~nm}$ and $90 \%$ transmittance at $510 \mathrm{~nm}$.

4.1.2. Spectral response. The spectral response of the fabricated diodes with on-chip filter was measured using the setup shown in figure 4(a), and the results are shown in figure 4(b). Light from a halogen lamp was tuned to a specific wavelength range using a monochromator. To measure the optical power differences among wavelengths, a calibrated photodiode (without any filter) was placed in front of the monochromator and the optical power corresponding to each wavelength was measured. Our on-chip photodiode (with an interference filter) was then placed in the light path and the diode response was measured for various wavelengths (450-700 nm). Normalized quantum efficiency of the diode was then calculated over the entire visual spectrum, by dividing the diode response by the appropriate optical power correction factors. From spectral response measurements shown in figure $4(b)$, we can observe that the normalized quantum efficiency (for filter + diode) is greater than $80 \%$ for wavelengths greater than $515 \mathrm{~nm}$. This is well suited for our fluorescence applications (excitation $491 \mathrm{~nm}$ : emission $511 \mathrm{~nm}$ ). Furthermore, the spectral response is unaffected by the process of depositing a $3 \mu \mathrm{m}$ thick layer of low temperature oxide on top of the filter. The LTO (or parylene-C) layer isolates the electronic component (diode) from the fluidic components and is necessary for any fluidic application. 
Detector calibration

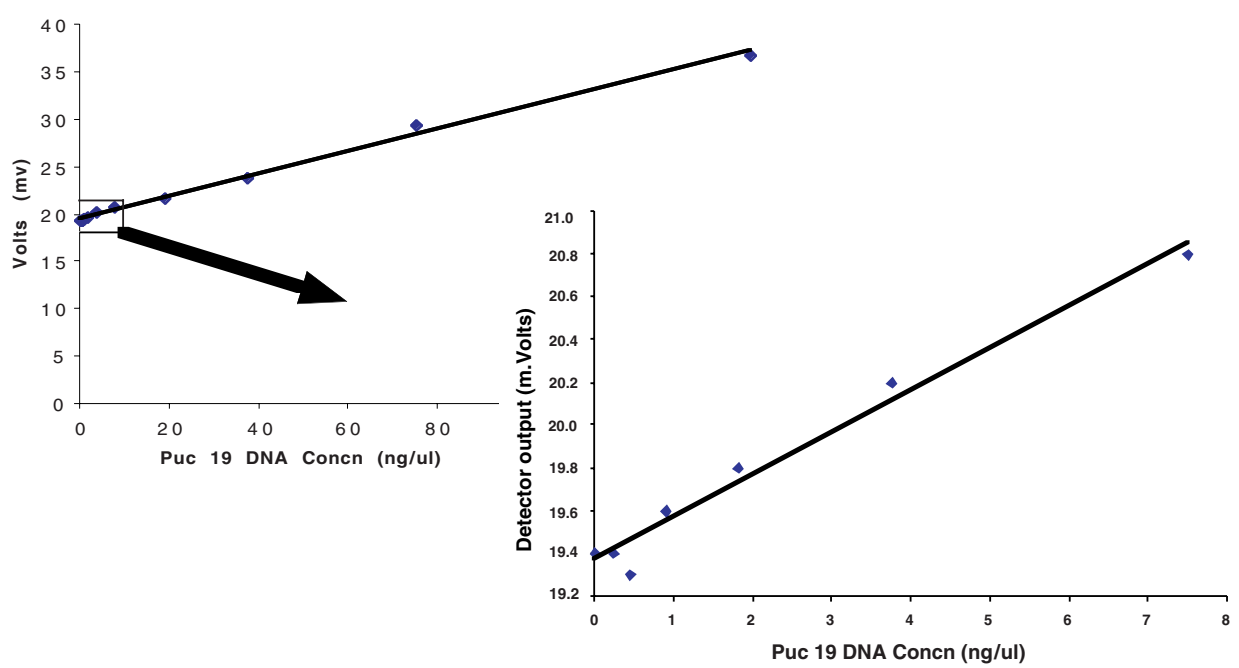

Figure 5. Response of the on-chip fluorescence detector to different DNA concentrations. Solution of varying concentrations of PUC 19 plasmid DNA (2.7 kbp) stained with YOYO-1 placed in a microfluidic device and the measurements are taken using the on-chip photodiode.

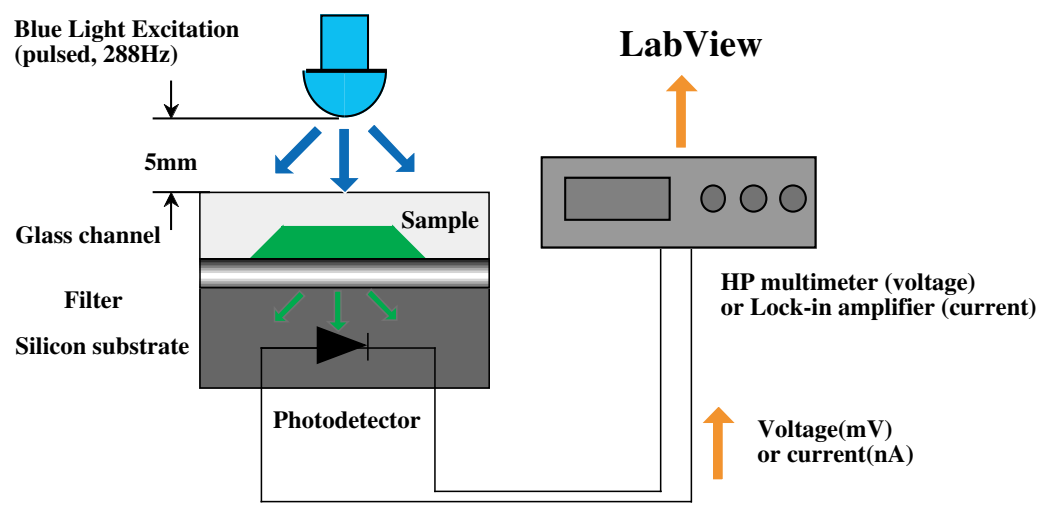

Figure 6. Experimental setup for on-chip fluorescence detection. Filtered (blue) light from a LED is used to excite DNA samples (stained with fluorescence dyes) inside a microfluidic channel. The fluorescent light signal is collected by the photodiode in the silicon substrate. Photoinduced voltages were measured using a HP multimeter that was interfaced to a computer with a LabView program.

4.1.3. Detection limits. Figure 5 shows the detector response as a function of DNA concentration in solution placed over the diode. This series of measurements was taken using a standard channel format (glass channel cap, $500 \mu \mathrm{m} \times 50 \mu \mathrm{m}$ cross-section), and a solution of $2.7 \mathrm{~kb}$ plasmid (pUC19, Gibco Life Technologies, Gaithersburg, MD) stained with YOYO-1 (Molecular Probes, Eugene, OR) dye. Similar experiments conducted in our earlier detector structure (figure 2(a)) yielded a sensitivity of $\sim 10 \mathrm{ng} \mu \mathrm{l}^{-1}$ (Burns et al 1998, Webster et al 2001). Our new design allows detection of DNA concentrations down to approximately $0.9 \mathrm{ng} \mu \mathrm{l}^{-1}$, showing an order of magnitude improvement over the previous design. Based on the size of the detector $(10 \mu \mathrm{m} \times 500 \mu \mathrm{m})$ and the volume of solution above it (50 $\mu \mathrm{m}$ high channel), this corresponds to a detection limit of approximately 5000 molecules. The linear nature of the calibration plot can be used to precisely quantify the concentration of DNA molecules present in solution.

\subsection{Applications}

The experimental setup for on-chip fluorescence detection is shown in figure 6. Filtered light from a blue LED or a mercury lamp is used for excitation. Two band-pass filters$485 \pm 11 \mathrm{~nm}$ and $450 \pm 30$ were used. With the first bandpass filter, the fluorescent dye (YOYO-1) is more efficient since we excite the dye at its excitation maximum. With the second filter, the on-chip filter is more efficient. Using the second filter we operate at wavelengths farther away from the edge of the on-chip filter and hence almost $99 \%$ of the excitation light is blocked from reaching the detector resulting in lower background signal. The second filter was found to be favorable in terms of signal-to-noise ratio of the diode and hence was used in most of our experiments. DNA tagged with fluorescent markers is placed within a microfluidic channel assembled on top of the diode substrate. The fluorescent light signal is collected by the photodiode in the silicon substrate. Photoinduced voltages were measured using a HP multimeter that was interfaced to a computer with a Labview program. Photoinduced currents were also measured using a lock-in amplifier that was interfaced to a computer with a Labview program. In the following sections, we describe the various applications of an on-chip photodiode in an integrated DNA analysis device. 


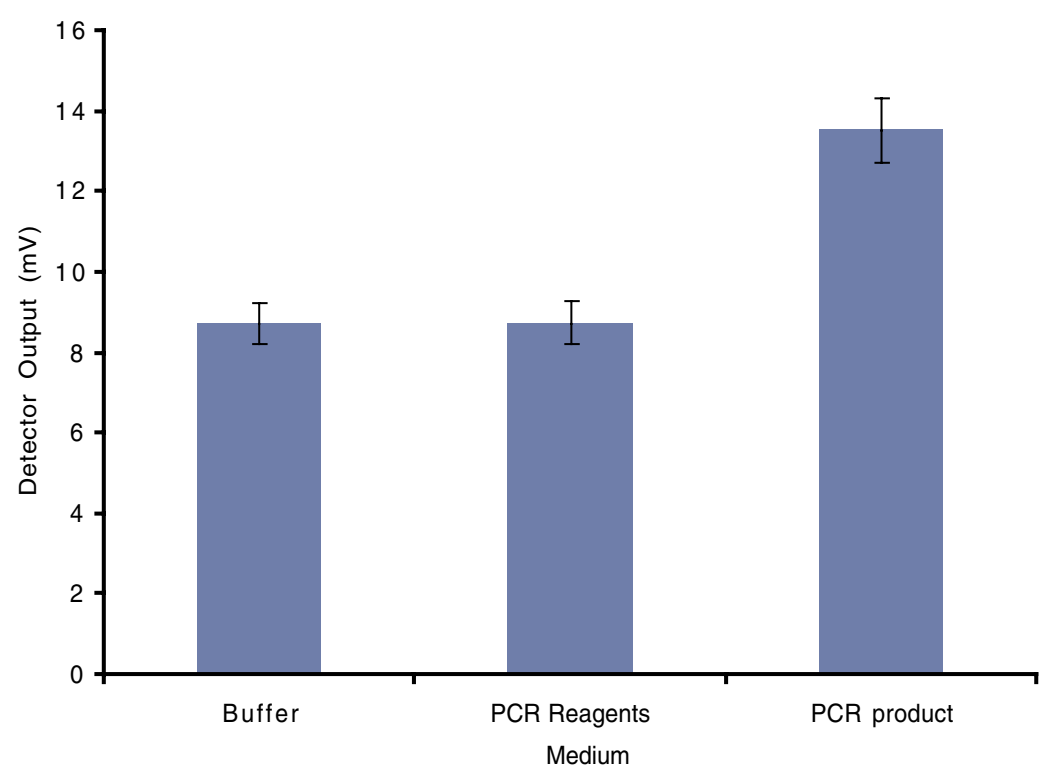

Figure 7. Data showing start and end points of a SYBR-PCR. A 1000 bp gene (Peg1 gene subcloned into the PGEM T-easy vector) was amplified using Taq polymerase. Reaction was done in the presence of SYBR Green $(1 \times$ concentration).

4.2.1. Microreactions. DNA amplifications using Polymerase chain reaction have evolved as an important analytical method in diagnostics for detection of disease causing pathogens, and in forensics for genetic profiling from trace amounts of sample (Erlich 1989). As a result, miniaturization of PCR chambers and detecting the amplified products using fluorescence measurements is a topic of significant interest (Kalinina et al 1997). Our photodiodes are extremely sensitive to very low concentrations of DNA

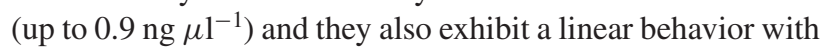
varying concentrations of DNA (figure 5).

This property can be exploited to monitor a PCR in real time or to quantify the amount of DNA at the end of a PCR. To demonstrate end-point detection capability, a target DNA (Peg1 gene subcloned into the PGEM T-easy vector) of size 1000 bp was PCR amplified using Taq polymerase in a commercial thermocycler. SYBR green (at $1 \times$ concentration), an intercalating dye was added to the PCR mix before the reaction (total reaction volume $20 \mu \mathrm{l}$ ). After 35 cycles, the products of the reaction were loaded in a microchannel placed over a set of photodiodes and the response was measured. Figure 7 shows the detector values at the start and end points of the SYBR-PCR.

These photodiodes can also be used for real time monitoring of PCR, where fluorescence data from the DNA are measured for a short time (typically $1 \mathrm{~s}$ ) at one specific temperature (extension phase) at the end of every cycle. Although PCR does take place over a $35^{\circ} \mathrm{C}$ range (60-95), the fluorescence data are always collected at one specific temperature. Hence, temperature dependence of these photodetectors will not be a significant factor and can therefore be used for monitoring fluorescence during PCR.

4.2.2. Microseparations. Separation resolution in miniaturized electrophoresis columns is also influenced by the size of the detector area. By having small area $(10 \mu \mathrm{m}$ wide) photodetectors in very close proximity to the separation column, detector related resolution losses can be minimized. Further, on-chip photodiodes coupled with LED-based excitation scheme would eliminate the need for an expensive laser-based confocal epi-fluorescence system. Our miniaturized electrophoresis system uses a simple crosschannel configuration to allow electrokinetic loading of DNA samples from the intersection area. We employed a uvpolymerizable cross-linked polyacrylamide gel as the sieving matrix (Reprogel). A flat gel interface could be defined at a precise location, using the photodefinable polacrylamide gel (Brahmasandra et al 2001). The DNA sample was compacted onto the interface by applying a low electric field $\left(6-8 \mathrm{~V} \mathrm{~cm}^{-1}\right)$ for $10 \mathrm{~s}$. Separation was done under constant electric field strength of $18.1 \mathrm{~V} \mathrm{~cm}^{-1}$.

We have used this gel electrophoresis system with onchip photodiodes for separation (with real time detection) of several DNA samples. For example, the PCR product that was analyzed in the previous (Microreactions) section (1000 bp long Peg1 gene) was loaded onto an electrophoresis column and separated. This was done to ensure the specificity of the reaction. Figure 8 shows a picture of the migrating PCR product as it passes over the diode and the corresponding detector output. Commercial samples (DNA ladders and plasmid digests) have also been analyzed using this setup. Figure 9 shows separation and detection of a commercially available 100 bp DNA ladder labeled with YOYO-1 intercalating dye. The first four bands (100$400 \mathrm{bp}$ ) were well-resolved close to gel interface and they were detected using a photodiode $4 \mathrm{~mm}$ from the interface. The remaining eight bands were resolved further down the electrophoresis column and detected using another photodiode $8 \mathrm{~mm}$ from the gel interface. On-chip photodetection gives us the power to incorporate several diodes along the length of the electrophoresis column and this offers the flexibility to use different diodes at appropriate locations to obtain maximal separation resolution. 
PCR Product - Separation and Detection

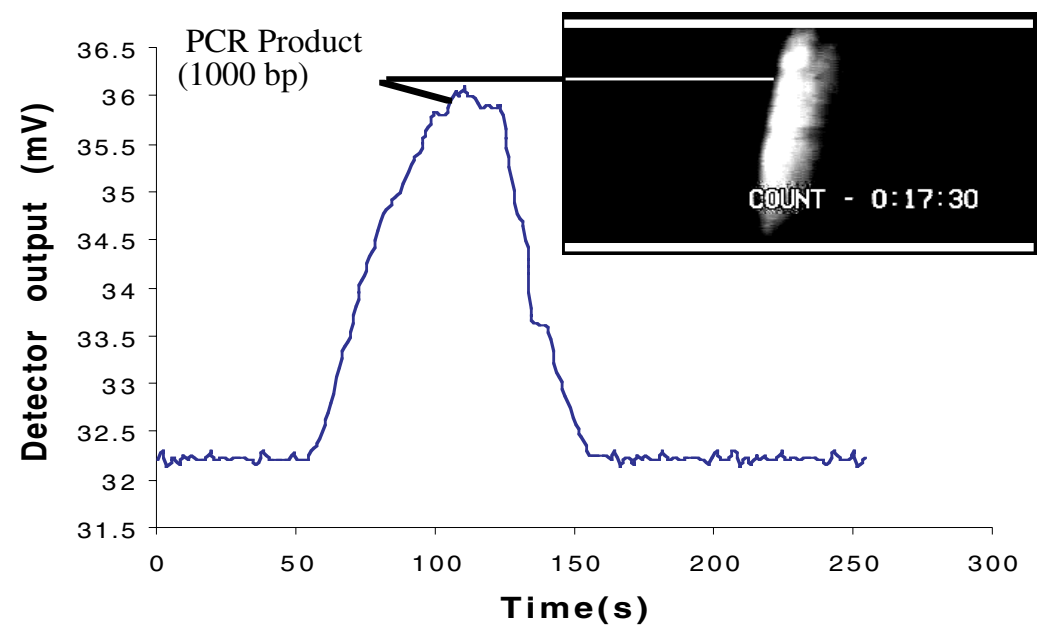

Figure 8. Detector output with video sequence depicting separation performance of PCR product (Sp1A 996 bp) under non-denaturing conditions using a commercial cross-linked polyacrylamide (ReproGel ${ }^{\mathrm{TM}}$ ) gel. Migration direction is left to right.

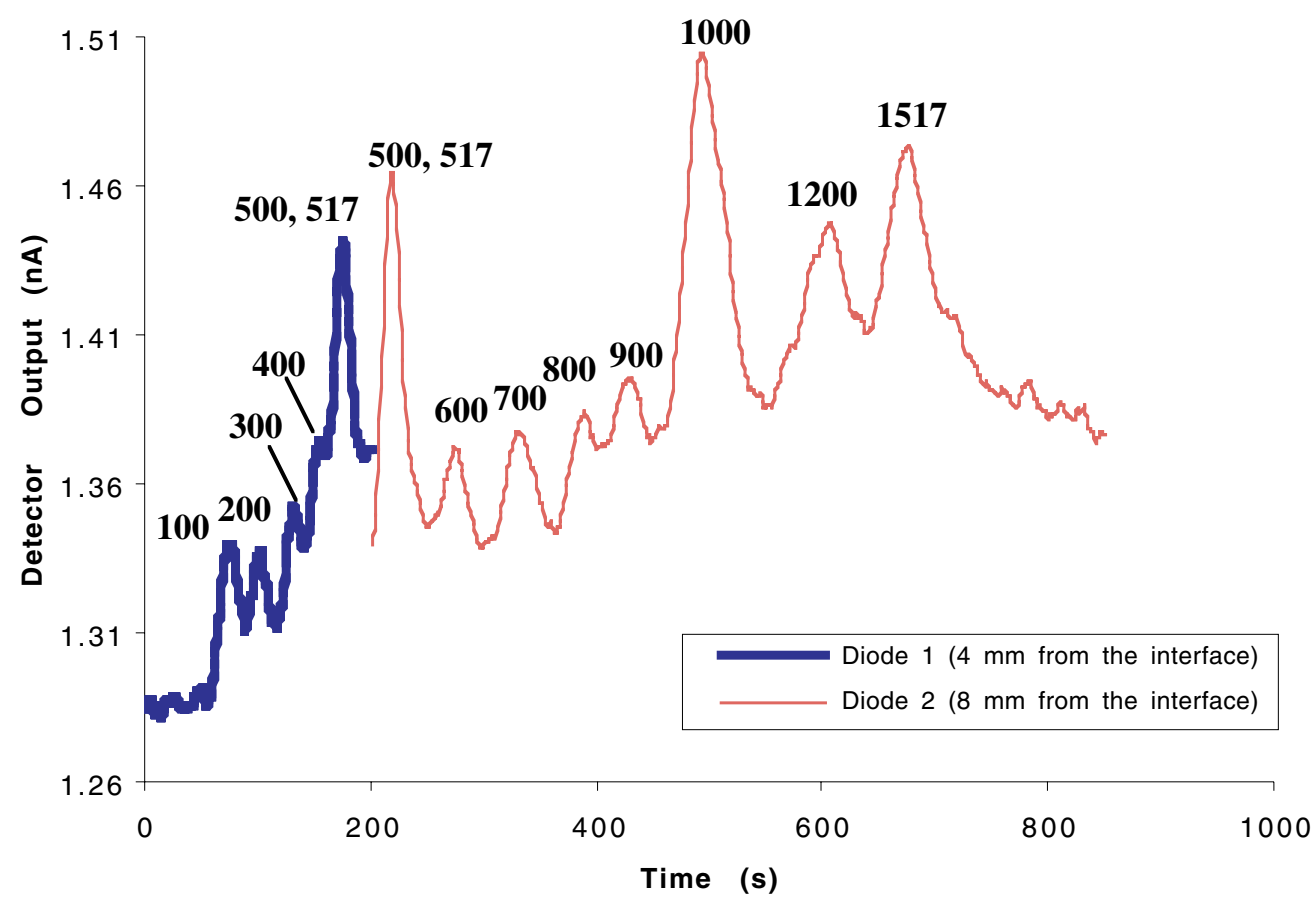

Figure 9. The electrophoretic separation and simultaneous on-chip detection of a commercially available 100 bp DNA ladder labeled with YOYO-1 intercalating dye. A flashing blue LED was used as the excitation source. The output is recorded from two diodes detector immediately beneath the electrophoresis channel. The solid thick (blue) line shows the response from the diode $4 \mathrm{~mm}$ from the gel interface. The first four bands $(100,200,300,400 \mathrm{bp})$ can be detected by this diode. The remaining eight bands are detected by another diode $8 \mathrm{~mm}$ far away from the gel interface, as shown by the thin (red) line. DNA fragment sizes in number of bases are denoted above each peak.

4.2.3. Microfluidics. In microfluidics, these photodiodes can be used to determine the position of a moving liquid droplet inside fluidic channels. When a liquid drop is positioned over the diode, the refractive index of the medium above the diode is higher as compared to the case when there is air over the diode. This change in refractive index changes the transmission qualities of the thin-layer optical filter on the device. Our drop detection principle is based on monitoring these changes in the transmission properties of the filter (figure 10(a)). The same blue light source used for fluorescence excitation can be used for drop position sensing.
When a drop moves over a diode, the change in the refractive index results in a decrease in the amount of incident light reaching the diode and the photoinduced voltage signal decreases. Once the drop moves away from the diode, more light reaches the diode and the signal increases (figure 10(b)). A series of diodes on the chip working on this principle can be used to electronically monitor the position of a moving droplet.

4.2.4. Temperature sensing. The diodes fabricated for sensing fluorescent light from DNA samples can also be used 
(a) Incident Light

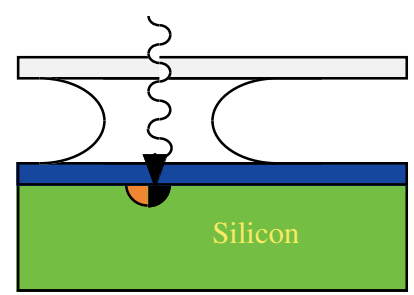

Liquid Drop

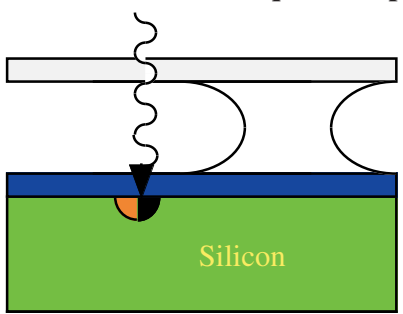

(b)

On Chip Drop Detection

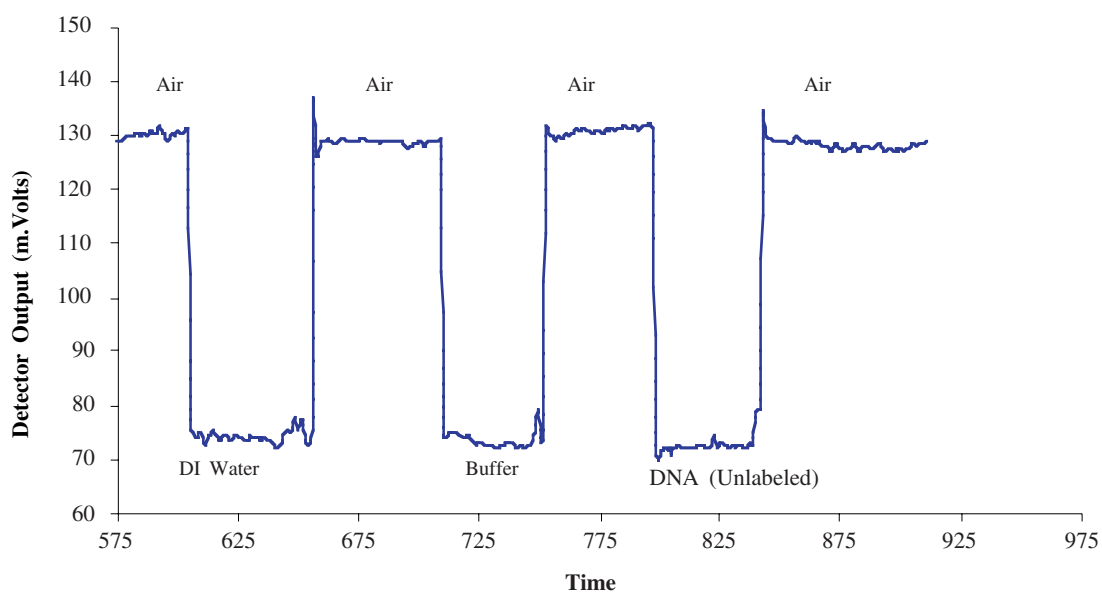

Figure 10. Data showing photovoltaic response of the diode when different liquid drops are positioned over the detector. The amount of light reaching the diode decreases when a liquid drop (higher refractive index than air) is over the diode causing a decrease in the detector response.

Temperature Response

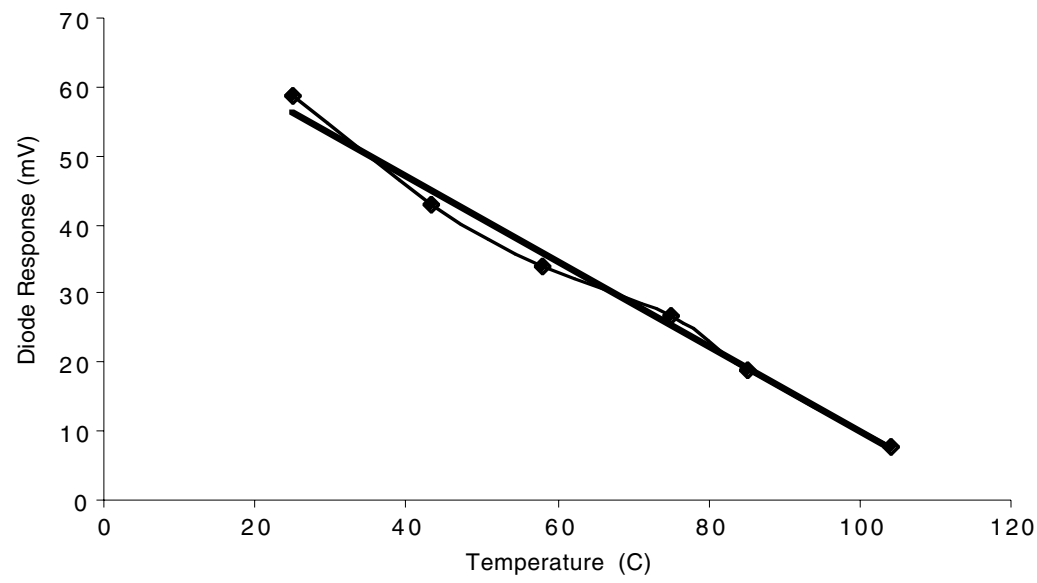

Figure 11. Data showing temperature response of the photodiode. Experiments were performed under constant background illumination (room light conditions). The temperature was measured using RTDs based on linear relationship between resistance and temperature.

as temperature sensors (Sclar 1972). The built in potential of the diode varies with temperature changes as given by the expression

$$
q V_{\mathrm{bi}}=E_{\mathrm{g}}-k T \ln \left(\frac{N_{\mathrm{c}} N_{\mathrm{v}}}{N_{\mathrm{n}} N_{\mathrm{p}}}\right)
$$

where $V_{\mathrm{bi}}$ is the built in potential, $E_{\mathrm{g}}$ is the band gap, $k$ is the Boltzmann constant, $T$ is the temperature. $N_{\mathrm{c}}, N_{\mathrm{v}}$ correspond to the density of states in the conduction band, valence band and $N_{\mathrm{n}}, N_{\mathrm{p}}$ correspond to the dopant density in the n-type, p-type region.

Figure 11 shows the typical response of these diodes to temperature changes. Since the diodes exhibit a linear temperature response, it is relatively easy to calibrate them. Further, when it comes to complex integrated genetic analysis devices, the diodes have two clear advantages over RTDs as temperature sensors. First, the active area of the diode is very small $(10 \mu \mathrm{m} \times 10 \mu \mathrm{m})$ which saves area on the chip. Second, they require no power input as compared to RTDs that require power to function. In addition, several diodes can be easily multiplexed to provide a complete thermal image of the entire device. However, the temperature sensing resolution of the diodes is currently at $0.5^{\circ} \mathrm{C}$ as compared to $0.1^{\circ} \mathrm{C}$ for RTDs.

\section{Conclusions}

Integration of on-chip photodiodes onto complex DNA analysis devices will aid in the development of practical hand-held diagnostic instruments. The on-chip photodiode structure $\left(\mathrm{PINN}^{+}\right)$presented in this work yields a DNA detection sensitivity of $0.9 \mathrm{ng} \mu \mathrm{l}^{-1}$, thus showing an order of improvement over earlier designs that can detect DNA concentrations only up to $10 \mathrm{ng} \mu \mathrm{l}^{-1}$. We have demonstrated applications of these on-chip photodiodes 
in several components of an integrated genetic analysis device; these applications include DNA concentration estimation in microreactors (PCRs), DNA band detection in microelectrophoresis columns, drop sensing in fluidic channels and temperature sensing.

Although PIN diodes presented in this work provide an excellent signal-to-noise ratio, the detection scheme can be improved further. First, PIN diodes do not have any inherent amplification. Avalanche photodiodes with internal amplification or PIN diodes connected to on-chip CMOS circuitry could be utilized for signal amplification. Second, the collinear illumination arrangement used in the present work allows some fraction of the incident light to reach the photodetector despite the presence of the on-chip filter. To improve the detector sensitivity, non-collinear illumination systems such as backside illumination schemes could be implemented. By adding some additional processing steps to fabricate these diodes, this on-chip detection scheme could be extremely valuable especially for constructing hand-held diagnostic instruments.

\section{References}

Barnes M D et al 1995 Detecting single molecules in liquids Anal. Chem. 67 418A-23A
Brahmasandra S N et al 2001 Electrophoresis in microfabricated devices using photopolymerized polyacrylamide gels and electrode-defined sample injection Electrophoresis 22 300-11

Burns M A et al 1998 An integrated nanoliter DNA analysis device Science 282 484-7

Chamberlain S G 1979 New profiled silicon photodetector for improved short-wavelength quantum efficiency IEEE Trans. Electron. Devices, J. Appl. Phys. 34 7228-31

Erlich H A 1989 PCR Technology: Principles and Applications for DNA Amplification (New York: Stockton Press)

Ewing A G et al 1994 Electrochemical detection in microcolumn separations Anal. Chem. 66 527a-535a

Hsue Y-T, Smith R L and Northrup M 1995 A microfabricated electrochemiluminescence cell for the detection of amplified DNA Proc. IEEE Conf. on Solid-State Sensors and Actuators pp 768-71

Hunkapiller T et al 1991 Large-scale and automated DNA sequence determination Science 254 59-67

Kalinina O et al 1997 Nanoliter scale PCR with TaqMan detection Nucl. Acids Res. 25 1999-2004

Metzker M et al 1996 Electrophoretically uniform fluorescent dyes for automated DNA sequencing Science 271 1420-2

Sclar N and Pollock D B 1972 On diode thermometers Solid-State Electron 15 473-80

Webster J R et al 2001 Monolithic capillary electrophoresis device with integrated fluorescence detector Anal. Chem. 73 1622-6

Wilson J and Hawkes J 1988 Optoelectronics: An Introduction (Englewood Cliffs, NJ: Prentice-Hall)

Woolley A T et al 1998 Capillary electrophoresis chips with integrated electrochemical detection Anal. Chem. 70 684-8 